\title{
SEGMENTATION USING THE EDGE STRENGTH FUNCTION AS A SHAPE PRIOR WITHIN A LOCAL DEFORMATION MODEL
}

\author{
Erkut Erdem* \\ Télécom ParisTech \\ CNRS UMR 5141 LTCI \\ Paris, France
}

\author{
Sibel Tari \\ Middle East Technical University \\ Dept. of Computer Engineering \\ Ankara, Turkey
}

\author{
Luminita Vese \\ University of California, Los Angeles \\ Department of Mathematics \\ Los Angeles, U.S.A.
}

\begin{abstract}
This paper presents a new image segmentation framework which employs a shape prior in the form of an edge strength function to introduce a higher-level influence on the segmentation process. We formulate segmentation as the minimization of three coupled functionals, respectively, defining three processes: prior-guided segmentation, shape feature extraction and local deformation estimation. Particularly, the shape feature extraction process is in charge of estimating an edge strength function from the evolving object region. The local deformation estimation process uses this function to determine a meaningful correspondence between a given prior and the evolving object region, and the deformation map estimated in return supervises the segmentation by enforcing the evolving object boundary towards the prior shape.
\end{abstract}

Index Terms - prior-based image segmentation, registration, variational methods

\section{INTRODUCTION}

The goal of segmentation is partitioning an image into coherent regions that are likely to correspond to objects which are imaged. Finding region boundaries accurately becomes particularly challenging when the corrupting influences due to missing regions, partial occlusions and noise appear in images. Recent works, including $[5,6,7,8,11,13,16]$, resolve these ambiguities by integrating lowlevel image features with high-level shape information. With the exception of [7], these works represent prior shape globally. In [7], Hong et al. present an alternative formulation which takes into account a local deformation model to constrain the shape of the evolving contour. The model captures a different appearance of the object of interest with ease by accordingly warping the reference shape. This, in a certain extent, provides an advantage over the global models.

In this paper, we employ the local deformation model of Hong et al. [7] in a new framework for prior-guided image segmentation. Specifically, our work differs from [7] in two aspects. The first difference is the way we formulate the segmentation energies. It is expressed as the minimization of three coupled functionals defining three processes that respectively account for prior-guided segmentation, shape feature extraction and local deformation estimation. The second difference is the way we represent the shape itself. As the experimental results demonstrate, our framework not only extracts the

\footnotetext{
* This study was completed when the author was at METU and at that time he was supported in part by TUBITAK-BAYG PhD scholarship.
}

object of interest from an image but also simultaneously registers the prior shape on the image data, allowing further semantic analysis to be performed on the extracted object.

\section{REPRESENTATION OF THE PRIOR SHAPE}

In our work, we represent the shape knowledge by a smooth edge indicator function $v$ called the edge strength function $[1,14]$ which is recently applied in prior-guided segmentation [6] in modeling the shape variability globally.

The edge strength function $v$ is the minimizer of

$$
\frac{1}{2} \int_{\Omega}\left(\rho|\nabla v|^{2}+\frac{(1-v)^{2}}{\rho}\right) d x
$$

where $\Omega \subset \mathbb{R}^{2}$ is an open, bounded and connected domain (the shape domain) with a boundary $\Gamma$. $v$ varies between 0 and 1 (s.t. $\left.v\right|_{\Gamma}=0$ ), and as $\rho \rightarrow 0, v \rightarrow 1$ everywhere except along $\Gamma$. Thus, the parameter $\rho$ may be interpreted as the blurring radius. In [15] the connections among edge strength function, curve evolution and diffused distance transform have been shown (see Fig. 1).

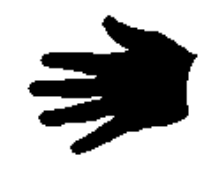

(a)

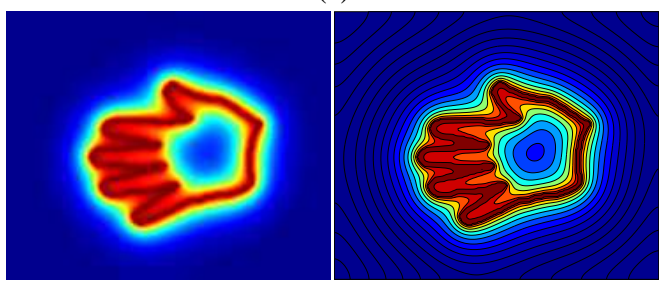

(b)

(c)

Fig. 1. The edge strength function $v$ reproduced using the method in [15]. (a) Binary silhouette. (b) $v$ computed with $\rho=8$. (c) Level curves of $v$. 


\section{ANALYSIS OF SHAPES USING A LOCAL DEFORMATION MODEL}

Shape matching plays a key role when analyzing the similarities between two given shapes by establishing a correspondence. In [7], Hong et al. suggest a local deformation model for shape matching. We adopt it in our formulation.

Let $v_{1}$ and $v_{2}$ denote the edge strength functions estimated from two silhouettes $S_{1}$ and $S_{2}$, respectively. Matching between the given shapes is estimated by minimizing the functional

$$
E_{\text {match }}(h)=E_{f i d}(h)+\beta E_{r e g}(h)
$$

with respect to the unknown deformation function $h: \Omega \rightarrow \mathbb{R}^{2}$. The first term in the energy $E_{f i d}(h)$ measures the similarity between the reference edge strength function and the template edge strength function which is transformed under the displacement vector field $h$.

In particular, the similarity term is defined as

$$
E_{f i d}(h)=\frac{1}{2} \int_{\Omega}\left(v_{2}(x+h(x))-v_{1}(x)\right)^{2} d x
$$

where $\Omega$ denotes the domain in which $v_{1}$ and $v_{2}$ are defined. While this term provides a driving force for the registration, the regularization term $E_{r e g}(h)$ forces the deformation field $h$ to be smooth. The relative importance between smoothing and similarity terms is determined by the parameter $\beta$.

In literature, several approaches are proposed to regularize the deformation field $h$. In our formulation, following the approach in [7], we employ the linear elasticity model proposed in [3] using a semi-implicit scheme for the time variable where the terms at the center pixel are treated implicitly while the remaining terms are treated explicitly:

$$
E_{r e g}(h)=\int_{\Omega}\left(\frac{\bar{\mu}}{4} \sum_{i, j=1}^{2}\left(\partial_{x_{i}} h_{j}+\partial_{x_{j}} h_{i}\right)^{2}+\frac{\lambda}{2}(\nabla \cdot h)^{2}\right) d x
$$

where $\partial_{x_{j}}$ denotes the partial derivative with respect to $x_{j}$, and $\lambda, \bar{\mu}>0$ are the Lamé constants that reflect material properties of an elastic body. This model cannot handle large deformations, and hence we assume that a global registration is initially performed.

The deformation field $h$ minimizing (2) formally satisfies the Euler-Lagrange equation

$$
\frac{\partial h}{\partial t}=-\frac{E_{m a t c h}}{\partial h}=-\left(\frac{\partial E_{f i d}}{\partial h}+\beta \frac{\partial E_{r e g}}{\partial h}\right)
$$

where

$$
\begin{aligned}
& \frac{\partial E_{f i d}}{\partial h}=\left(v_{2}(x+h(x))-v_{1}(x)\right) \nabla v_{2}(x+h(x)), \\
& \frac{\partial E_{r e g}}{\partial h}=-\left(\bar{\mu} \nabla^{2} h+(\lambda+\bar{\mu}) \nabla(\nabla \cdot h)\right) .
\end{aligned}
$$

In Fig. 2, we present an example matching result obtained by the proposed method. As it can be clearly seen, the resulting registration process determines a meaningful correspondence between the given silhouettes.
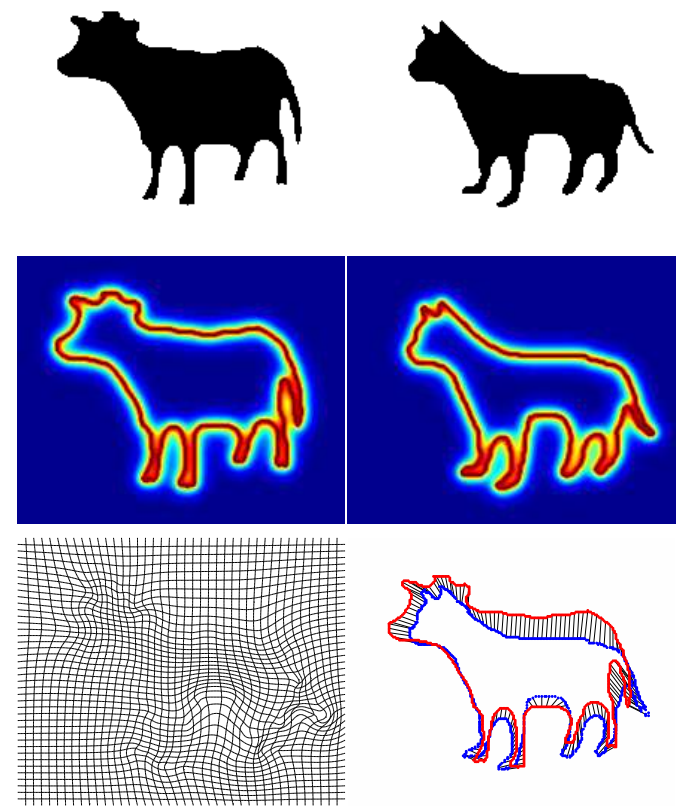

Fig. 2. Matching example. (top row) cow and cat silhouettes (adapted from [2]). (middle) Corresponding edge strength functions estimated with $\rho=4$. (bottom) Estimated deformation vector field and the matching result.

\section{SEGMENTATION FRAMEWORK}

In the previous section, a shape matching framework is developed by using a local deformation model. Now we will discuss how this model is employed within a segmentation framework to impose a priori shape information about an object of interest on the ongoing segmentation process.

We formulate image segmentation by minimization of three coupled functionals, $E_{1}, E_{2}$, and $E_{3}$ defined as follows:

$$
\begin{aligned}
E_{1}(\phi \mid h) & =E_{C V}(\phi)+\mu_{1} E_{\text {shape }}(\phi \mid h), \quad \mu_{1} \geq 0, \\
E_{2}(v \mid \phi) & =E_{\text {feature }}(v \mid \phi), \\
E_{3}(h \mid v, \phi) & =E_{\text {match }}(h \mid v, \phi) .
\end{aligned}
$$

Each functional has a distinct role in the overall process. The first functional $E_{1}$ is an extended version of the functional in [4]

$$
\begin{gathered}
E_{C V}(\phi)=\int_{\Omega}\left(f(x)-c_{1}\right)^{2} H(\phi(x)) d x \\
+\int_{\Omega}\left(f(x)-c_{2}\right)^{2}(1-H(\phi(x))) d x+\mu \int_{\Omega}|\nabla H(\phi(x))| d x
\end{gathered}
$$

with an additional prior shape term

$$
E_{\text {shape }}(\phi \mid h)=\int_{\Omega}(H(\phi(x))-T(x+h(x)))^{2} d x
$$

and mainly accounts for the prior-guided segmentation.

From computational point of view the functional (11), which is a well-known approximation for the Mumford-Shah segmentation model [9], follows a level-set based curve evolution formulation [10]. In (11), $\phi$ represents a level set function whose zero-level line corresponds to the contour segmenting the input image $f, H$ is 
a Heaviside function, $c_{1}$ and $c_{2}$ respectively denote the average gray values of foreground and background regions, and $\lambda_{1}, \lambda_{2}>0$ and $\mu \geq 0$ are fixed parameters. The prior shape term (12) is similar to the one used in [12] and constrains the shape of the evolving foreground/object regions by making use of a binary template $T$ given a priori. Mainly, it measures the difference in the areas of the evolving object region represented by $H(\phi)$ and the prior $T$ transformed under the deformation $h$. As a result, while (11) leads to an image force that attracts the zero-level curve of the evolving level set function $\phi$ to object boundaries, the prior term enforces the zero-level curve towards the given template $T$ under transformation.

The second functional $E_{2}$ is utilized for extracting shape features from the image regarding the object to be segmented and defined by

$$
\begin{aligned}
E_{\text {feature }}(v \mid \phi) & =\alpha \int_{\Omega} v^{2}|\nabla H(\phi)|^{2} d x \\
& +\frac{1}{2} \int_{\Omega}\left(\rho|\nabla v|^{2}+\frac{(1-v)^{2}}{\rho}\right) d x .
\end{aligned}
$$

In fact, (13) is a modified version of the functional in [15] where the fidelity term is excluded and the gray value image is replaced with $H(\phi)$. As a result, the edge strength function $v$ denoting the minimizer of (13) represents the shape of the evolving object region.

The third functional $E_{3}$ corresponds to the matching energy defined in (2) and responsible for estimation of the local deformation. With fixed $v$, minimizing this functional with respect to the unknown variable $h$ establishes a correspondence between the evolving object region and the prior shape that are respectively represented by the edge strength functions $v$ and $v_{T}$.

The functionals $E_{1}-E_{3}$ are coupled in the sense that the deformation field $h$ found by (10) determines the level set $\phi$; the level set $\phi$ estimated by (8) specifies the edge strength function $v$, and $v$ defines the deformation field $h$. These functionals work jointly to partition an image into object vs. background regions.

Our strategy is to alternate between these functionals when we apply the gradient descent. We fix the deformation field $h$ when we try to minimize the first functional $E_{1}$, and determine the level set $\phi$. Similarly, we fix the level set function $\phi$ when we try to minimize the second functional $E_{2}$, and estimate the evolving shape represented by the edge strength function $v$. After that, with fixed $v$ we estimate the deformation field $h$. Note that we could have combined these functionals and have started with it, interpreting it as a general framework. The main disadvantage of such a formulation is that when we apply the gradient descent, the deformation field $h$ depends on not only the edge strength functions, but also the binary silhouettes, which may yield inaccurate correspondences.

We now apply gradient descent to the functionals $E_{1}-E_{3}$ to obtain segmentation. Minimizing the first functional (8) with respect to $\phi$ leads to the following evolution equation:

$$
\frac{\partial \phi}{\partial t}=-\frac{E_{1}}{\partial \phi}=-\left(\frac{E_{C V}}{\partial \phi}+\mu_{1} \frac{E_{\text {shape }}}{\partial \phi}\right)
$$

with

$$
\begin{aligned}
\frac{E_{C V}}{\partial \phi} & =\delta(\phi)\left[\left(f-c_{1}\right)^{2}-\left(f-c_{2}\right)^{2}-\mu \nabla \cdot\left(\frac{\nabla \phi}{|\nabla \phi|}\right)\right], \\
\frac{E_{\text {shape }}}{\partial \phi} & =2 \delta(\phi(x))(H(\phi(x))-T(x+h(x))) .
\end{aligned}
$$

With fixed $\phi$, minimizing the second functional (9) with respect

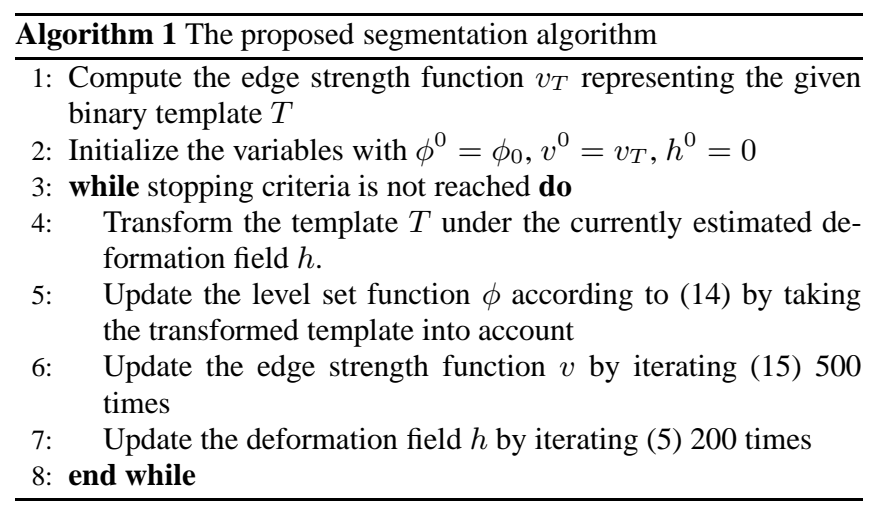

to $v$ leads to the evolution equation

$\frac{\partial v}{\partial t}=-\frac{E_{2}}{\partial v}=-\frac{E_{\text {feature }}}{\partial v}=-\nabla^{2} v+\frac{2 \alpha|\nabla H(\phi)|^{2} v}{\rho}+\frac{(v-1)}{\rho^{2}}$

Finally, the evolution equation for $h$ obtained from the third functional is similar to (5) where $v_{2}$ is replaced with $v_{T}$, and $v_{1}$ with $v$. The whole minimization procedure of the proposed framework is summarized in Algorithm 1. In the experiments, the iterations continue until the overall energy $E_{1}+E_{2}+E_{3}$ is no more decreasing.

In Fig. 3, we present sample segmentation results on various natural images. In order to demonstrate the performance of our framework and to illustrate what it is gained by introducing prior shape knowledge, we compare the results of our method with those of the model in [4] using the same values for the common parameters and the same initial conditions. Note that setting $\mu_{1}$ to zero and excluding $E_{2}$ and $E_{3}$ from computations reduce our formulation to the model in [4], yielding a model that does not take prior shape into account. In all experiments, we set $\rho=4, \alpha=500, \beta=0.01$, $\lambda=0, \bar{\mu}=1$ unless stated otherwise, and assume that a global registration is initially performed. An advantage of our framework is that it performs image segmentation with simultaneously registering the prior shape on the image data. Fig. 4 presents a joint segmentation and registration result on a partially occluded horse image. The initial zero-level curve is broken into meaningful parts (head, tails, legs, etc.) and transformed under the estimated displacement vector field accordingly.

\section{SUMMARY}

We have presented a new prior-shape based image segmentation framework using the edge strength function within a local deformation model. The edge strength function has been previously used in prior-guided segmentation with a global deformation model. The prior shape information explicitly imposes higher-level influences on the ongoing segmentation process, yielding robustness against undesirable conditions such as partial occlusions and noise. Furthermore, the local deformation model determines a meaningful correspondence between a given template and the evolving object region, and leads to joint registration results. Due to this jointly performed registration process, our framework allows to perform additional semantic analysis as well when a shape analysis carried out beforehand. 

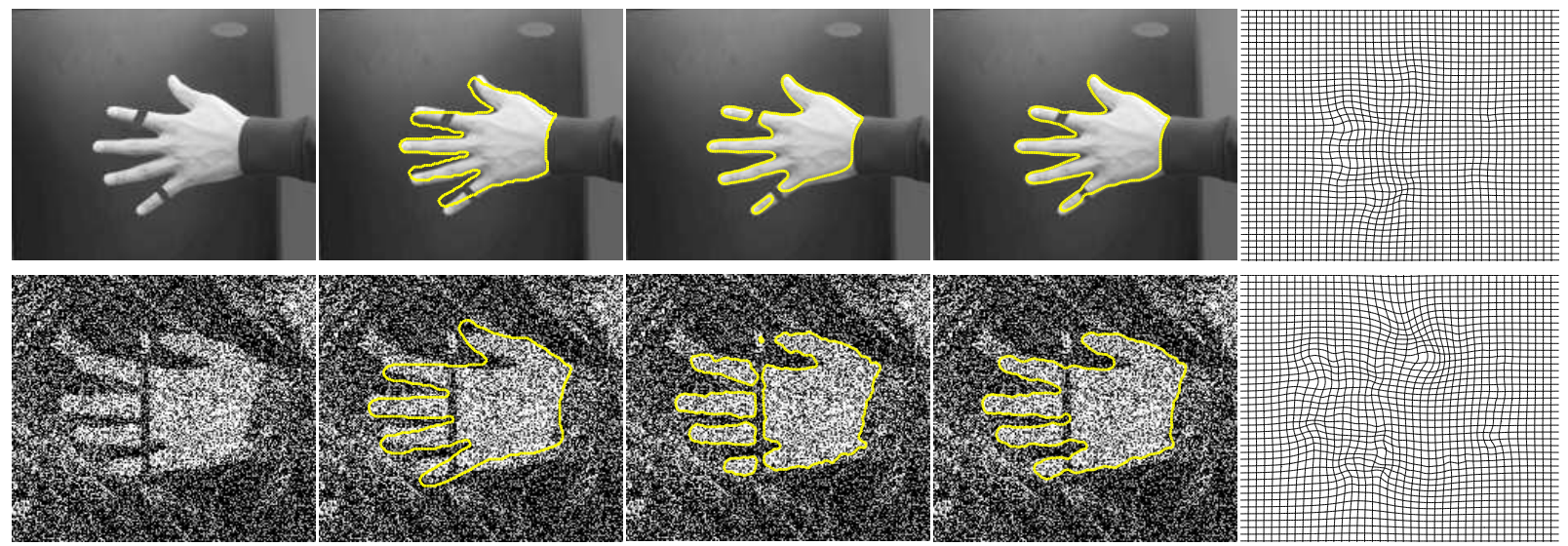

Fig. 3. Segmenting corrupted hand images with partial occlusions and significant amount of noise. (first column) Input images - top image is from [7]. (second) Unregistered prior shapes superimposed on the input images (initial zero-level curves). (third) Segmentation results without prior shape information. (fourth) Final segmentation results. (fifth) Deformed grids under the estimated displacement vector fields. (the parameters are $\mu_{1}=17500, \beta=0.025$ (top row), $\mu_{1}=12500$ (bottom row) and $\mu=20000$ ).
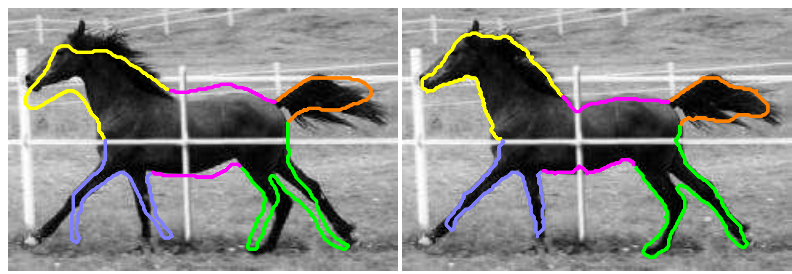

Fig. 4. Analysis of the registration process. Different colors are used for different parts of the segmenting contour in order to show how meaningful the obtained correspondences are (the parameters are $\mu=18000$ and $\mu_{1}=20000$ ).

\section{REFERENCES}

[1] L. Ambrosio and V. Tortorelli. On the approximation of functionals depending on jumps by elliptic functionals via $\Gamma$ convergence. Commun. Pure Appl. Math., 43(8):999-1036, 1990.

[2] X. Bresson, P. Vandergheynst, and J.-P. Thiran. A variational model for object segmentation using boundary information and shape prior driven by the Mumford-Shah functional. Int. J. Comput. Vision, 68(2):145-162, 2006.

[3] C. Broit. Optimal Registration of Deformed Images. PhD thesis, Computer and Information Science, University of Pennsylvania, 1981

[4] T. F. Chan and L. A. Vese. Active contours without edges. IEEE Trans. Image Processing, 10(2):266-277, 2001.

[5] D. Cremers, F. Tischhäuser, J. Weickert, and C. Schnörr. Diffusion snakes: Introducing statistical shape knowledge into the Mumford-Shah functional. Int. J. Comput. Vision, 50(3):295313, 2002.

[6] E. Erdem, A. Erdem, and S. Tari. Edge strength functions as shape priors in image segmentation. In EMMCVPR, pages 490-502, 2005.

[7] B.-W. Hong, E. Prados, S. Soatto, and L. Vese. Shape represen- tation based on integral kernels: Application to image matching and segmentation. In CVPR, pages 833- 840, 2006.

[8] M. E. Leventon, E. L. Grimson, and O. D. Faugeras. Statistical shape influence in geodesic active contours. In $C V P R$, pages 1316-1323, 2000.

[9] D. Mumford and J. Shah. Optimal approximations by piecewise smooth functions and associated variational problems. Commun. Pure Appl. Math., 42(5):577-685, 1989.

[10] S. Osher and J. A. Sethian. Fronts propagating with curvaturedependent speed: Algorithms based on Hamilton-Jacobi formulation. J. Comput. Phys., 79:12-49, 1988.

[11] T. Riklin-Raviv, N. Kiryati, and N. Sochen. Prior-based segmentation and shape registration in the presence of perspective distortion. Int. J. Comput. Vision, 72(3):309-328, 2007.

[12] T. Riklin-Raviv, N. Kiryati, and N. A. Sochen. Unlevel-sets: Geometry and prior-based segmentation. In ECCV, volume 4, pages 50-61, 2004.

[13] M. Rousson and N. Paragios. Shape priors for level set representations. In ECCV, volume 2, pages 78-92, 2002.

[14] J. Shah. Segmentation by nonlinear diffusion, II. In $C V P R$, pages 644-647, 1992.

[15] S. Tari, J. Shah, and H. Pien. Extraction of shape skeletons from grayscale images. CVIU, 66(2):133-146, 1997.

[16] A. Tsai, A. Yezzi, W. Wells, C. Tempany, D. Tucker, A. Fan, E. Grimson, and A. Willsky. A shape-based approach to the segmentation of medical imagery using level sets. IEEE Trans. Med. Imaging, 22(2):137-154, 2003. 